Veritas 日 Scientia

Vol. 9. $\mathrm{N}^{\circ} 1$

Enero - Junio del 2020

ISSN Edición Online: 2617-0639

https://doi.org/10.47796/ves.v9i1.281

\title{
LESIONES ATEROMATOSAS EN ARTERIAS CARDIOVASCULARES Y CEREBRALES EN POBLACIÓN ADOLESCENTE Y ADULTO JOVEN (POST MORTEM). REGIÓN DE TACNA, PERÚ
}

\author{
ATEROMATIC INJURIES IN CARDIOVASCULAR AND BRAIN ARTERIES IN ADOLESCENT POPULATION \\ AND YOUNG ADULT (POST MORTEM). TACNA REGION, PERU
}

\author{
Victor Arias Santana ${ }^{1}$ \\ Pamela Cáceres Cáceres ${ }^{2}$ \\ Sissy Mena Ordoñez ${ }^{3}$
}

Aceptado:01/07/2020

Publicado online:10/07/2020

\begin{abstract}
RESUMEN
Establecer una base del estado actual de daño en arterias mediante clasificación patológica en muestras post mortem.

Objetivo: Observar y comparar los cambios estructurales en las arterias aorta, cardiovascular carótida, y coronaria y cerebrales basilar posteriores, en cadáveres de personas de 15 a 35 años.

Método: Estudio observacional y analítico, prospectivo de corte transversal en el campo de la salud pública. Se trabajó con 24 cadáveres según criterios de inclusión y exclusión. Se clasificó el daño arterial según se el sistema de la American Heart Association.

Resultados: En aorta, las lesiones fueron de tipo I (45.8\%), 8.3\% tuvo una lesión tipo II, III, IV, respectivamente. En el de 15 a 19 años, el $60 \%$ presentaba lesiones tipo I. En carótida, el $62.5 \%$ tuvo una lesión de tipo I y un $12.5 \%$ lesión tipo II. El $100 \%$ del grupo de 15 a 19 años tenía una lesión tipo I en carótida. En arteria coronaria, un $75 \%$ tuvo lesiones tipo I, un $12.5 \%$ con lesiones tipo II. En el grupo de 15 a 19 años ninguna muestra coronaria era sana. En arteria basilar, el $33.3 \%$ tuvo lesiones tipo I, siendo más frecuente en el grupo de 20 a 29 años (44.4\%). En general, las lesiones tipo I se observó más en coronaria $(72 \%)$, las de tipo II coronaria y carótida (12\%), tipo III en aorta (8\%) y tipo IV en aorta (8\%).

Conclusión: Los daños estructurales en arterias empiezan en edades tempranas y se diferencian según localización arterial.
\end{abstract}

Palabras clave: ateroma, arteriosclerosis, aorta, carótida, basilar, coronarias.

\footnotetext{
1Doctor en Ciencias. Docente Universidad Privada de Tacna. sicuany@hotmail.com. ORCID: 0000-0001-9193-9918 2 Médico Jefe de la División Médico Legal II Tacna. Docente de la Universidad Privada de Tacna en cátedra medicina y anatomía humana. cacerescaceres_pamela@ hotmail.com. ORCID: 0000-0003-4221-6582 ${ }^{3}$ Magíster en Investigación. Docente de la Universidad Privada de Tacna. Especialista en Informática y Metodología Investigativa. sissymena@hotmail.com. ORCID:0000-0001-5950-5888
} 


\begin{abstract}
To establish a basis for the current state of damage to arteries through pathological classification in post-mortem samples.

Objective: To observe and compare the structural changes in the aortic, cardiovascular carotid, and coronary and posterior basilar cerebral arteries, in corpses of people aged 15 to 35 years.

Method: Observational and analytical, prospective cross-sectional study in the field of public health. We worked with 24 bodies according to inclusion and exclusion criteria. Arterial damage was classified according to the American Heart Association.

Results: In the aorta, the injuries were type I (45.8\%), 8.3\% had a type II, III, IV injury, respectively. In the 15-19 age group, $60 \%$ had type I injuries. In the carotid, $62.5 \%$ had a type I injury and $12.5 \%$ a type II injury. $100 \%$ of the group aged 15 to 19 had a type I carotid injury. In the coronary artery, $75 \%$ had type I injuries, $12.5 \%$ with type II injuries. In the group of 15 to 19 years, no coronary sample was healthy. In the basilar artery, $33.3 \%$ had type I lesions, being more frequent in the group of 20 to 29 years (44.4\%). In general, type I lesions were observed more in the coronary (72\%), type II coronary and carotid (12\%), type III in the aorta (8\%) and type IV in the aorta (8\%).

Conclusion: Structural damage to arteries begins at an early age and differs according to arterial location.
\end{abstract}

Key words: atheroma, arteriosclerosis, aorta, carotid, basilar, coronary.

\title{
INTRODUCCIÓN
}

La arteriosclerosis es la forma más común de daño vascular pero la que pasa más desapercibida. Si bien su daño más serio es el infarto de miocardio, se sabe que está ligada a muchos otros daños asociados a hipoxia celular. Como enfermedad crónica, generalmente se la vincula a población mayor, pero no existen trabajos locales ni nacionales que establezcan su estado en población adolescente o adulto joven. Cuanto más precoz es su presencia mayor es el daño a lo largo del tiempo en la mayoría de los órganos blanco. Si bien los estilos de vida poco saludables, la presencia de hábitos nocivos y enfermedades concomitantes como la diabetes están íntimamente ligados a su presencia, esto a la vez nos hace reflexionar que los mecanismos de prevención es la mejor arma para mitigar el daño en edad temprana. Se plantea estudiar los cambios estructurales en las arterias cerebrales basilar y arterias cerebrales posteriores, arterias cardiovasculares carótida Izquierda, coronaria izquierda y bifurcación de Aorta como principales marcadores en cadáveres de 15 a 35 años de una morgue local y establecer las características topográficas.

La aterosclerosis es la principal causa de morbimortalidad en la sociedad actual, y origina afecciones tales como el infarto agudo de miocardio (IAM), el accidente cerebrovascular (ACV), la enfermedad aneurismática y el síndrome de isquemia crónica de las extremidades. Causa el $50 \%$ de la mortalidad global en EE.UU y Europa occidental, (1) Estudios revelan que la edad es la principal variable asociada a arteriosclerosis media y avanzada(2) (3) (4). Pero es también claro reconocer que no existen trabajos, al menos en nuestro medio, en niños o jóvenes. El escaso recurso para el trabajo en vivos dificulta aún más el problema. Estudios 
post mortem, es un recurso indirecto que iniciaría una nueva línea de investigación regional basado en evidencia, especialmente en menores de 35 años

\section{METODOLOGÍA}

Observacional, analítico y prospectivo de corte transversal en el campo del análisis patológico y de salud pública. Se trabajó con el $100 \%$ de muestras post mortem que cumplieron los criterios de inclusión y exclusión de arterias cerebrales: Basilar, Cardiovasculares: Bifurcación de Aorta, Carótida Izquierda y Coronaria izquierda, procedentes de estructuras post mortem de 11 a 35 años, con integridad de las arterias, cualquier causa de muerte. El estudio analizó 24 personas en condición de post mortem, con el evento de fallecimiento dentro de las primeras 24 horas. Las muestras fueron analizadas en el instituto de Medicina legal. Los cortes histológicos fueron procesados y leídos a doble ciego por un patólogo. Se accedió a cinco muestras por caso. Las placas ateromatosas, se clasificaron de acuerdo con el sistema de la American Heart Association.

\section{RESULTADOS}

Se pudo evidenciar que el $41.7 \%$ de las personas fallecidas tenía entre 30 a 39 años seguido de un $27.5 \%$ entre 20 y 29 años y un $20.8 \%$ entre 15 a 19 años (ciclo de vida adolescente). Según sexo el $70.8 \%$ eran varones y el $29.2 \%$ mujeres.

Los resultados se presentan según estructura analizada.

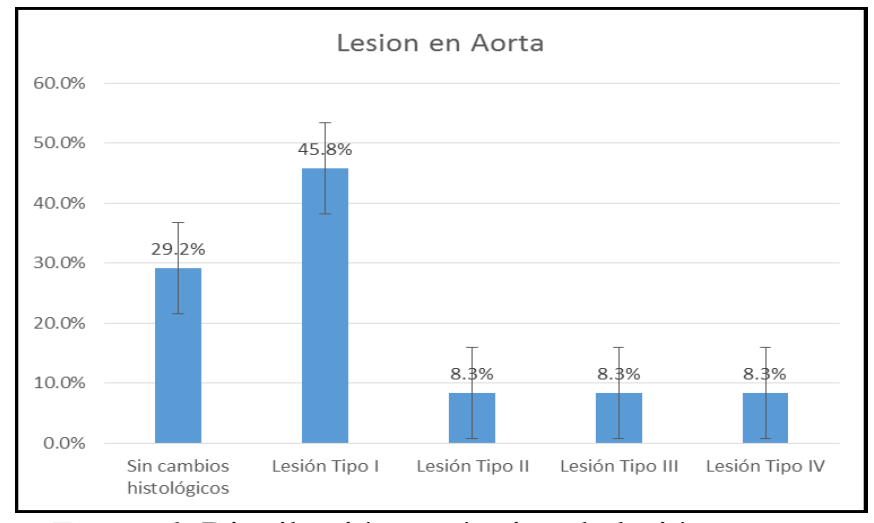

Figura 1. Distribución según tipo de lesión en aorta

Luego del análisis anatomopatológico, se pudo observar que las lesiones predominantemente presentes en aorta fueron las de tipo I (45.8\%). Un 8.3\% tuvo incluso lesión tipo IV (Fig. 1) Según edad, en el grupo de personas entre 15 a 19 años ya el 60\% presentaba lesiones tipo I en aorta. Estas proporciones según tipo de lesión fueron aumentando conforme la edad aumentaba, encontrándose un $55.6 \%$ con lesiones tipo I en grupo de 20 a 29 años, pero un $11.1 \%$ ya con lesión tipo II. En el grupo de 30 a 39 años se encontraron un $30 \%$ con lesiones tipo I, pero ya se observaron un $20 \%$ como lesiones tipo III y un $20 \%$ similar conexiones tipo IV. (Fig. 2) 


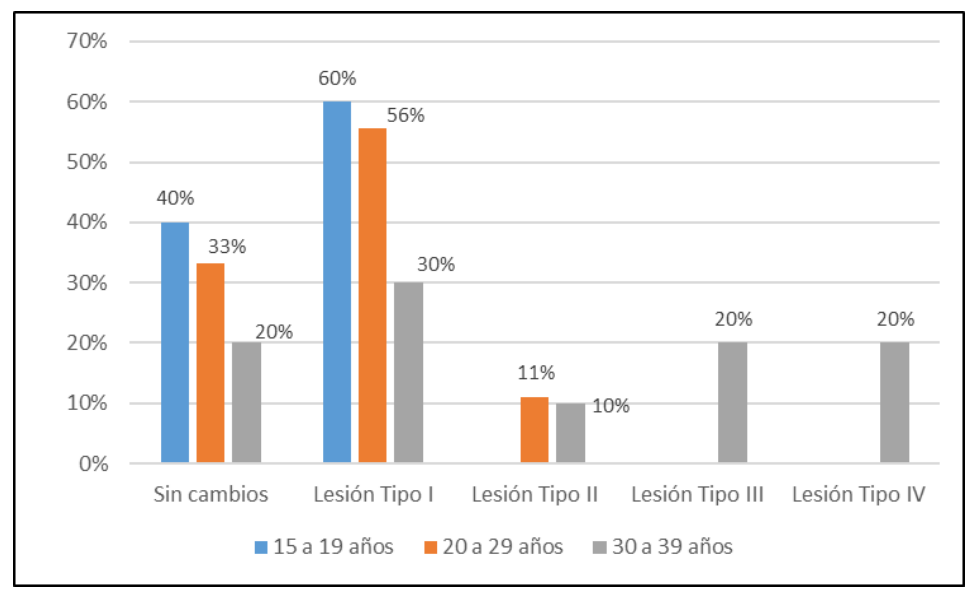

Figura 2. Distribución de lesión en aorta según edad

Podemos observar una presencia precoz de lesiones ateromatosas en la población en estudio que ameritaría un estudio mucho más amplio en poblaciones más grandes, puesto que sería la evidencia para cambiar las estrategias y estilos de vidas vinculado a este tipo de lesiones y que están arraigados en las costumbres de esta población.

Se analizó la lesión presente en carótida, observándose que el $62.5 \%$ de la población en estudio tuvo una lesión de tipo I y un $12.5 \%$ de lesión tipo II. Se observó también en menor proporción, pero presentes, lesiones tipo III en un $4.2 \%$ y lesiones tipo IV en $4.2 \%$. (Fig. 3 )

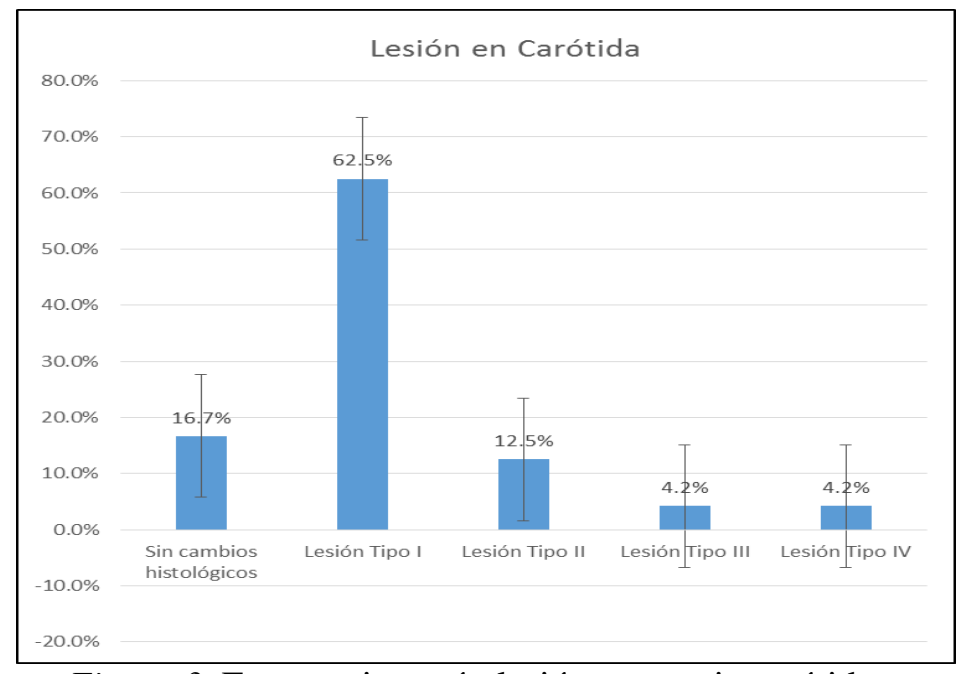

Figura 3. Frecuencia según lesión en arteria carótida.

Según edad, en el grupo de 15 a 19 años, las lesiones tipo I representaron el $100 \%$ de los casos. En el grupo de 20 a 29 años el $66.7 \%$ de las lesiones fueron de I y el $22.2 \%$ tipo II. En el grupo de 30 a 39 años se llegó a observar lesiones hasta de tipo IV con predominio de la lesión tipo I en un $40 \%$. 


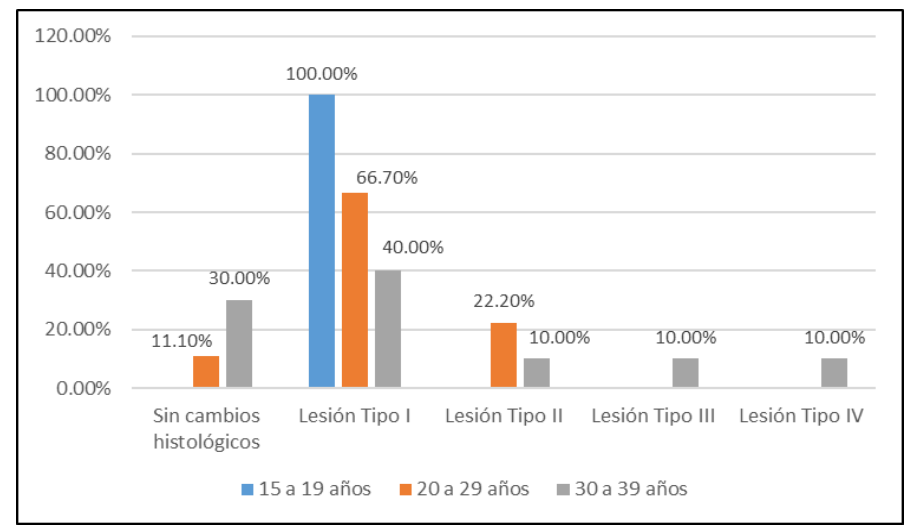

Figura 4. Frecuencia de lesión en arteria carótida según edad.

Podemos observar que esta ubicación es una de las más afectadas desde temprana edad. La presencia de lesiones en carótida afectó a todo el grupo de adolescente.

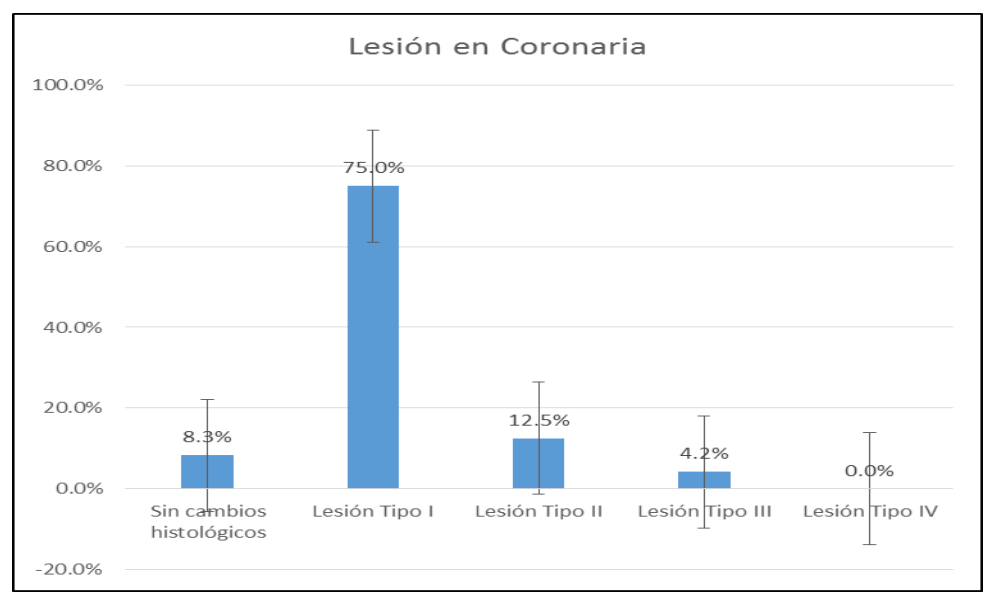

Figura 5. Frecuencia según tipo de lesiones en arteria coronaria.

Las arterias coronarias, son los principales predictores de calidad de estado de conservación de las arterias en el organismo. Se puedo evidenciar que un $75 \%$ tienen lesiones tipo I seguido de un $12.5 \%$ con lesiones tipo II. Una escasa proporción del $8.3 \%$ no presentaban cambios histológicos.

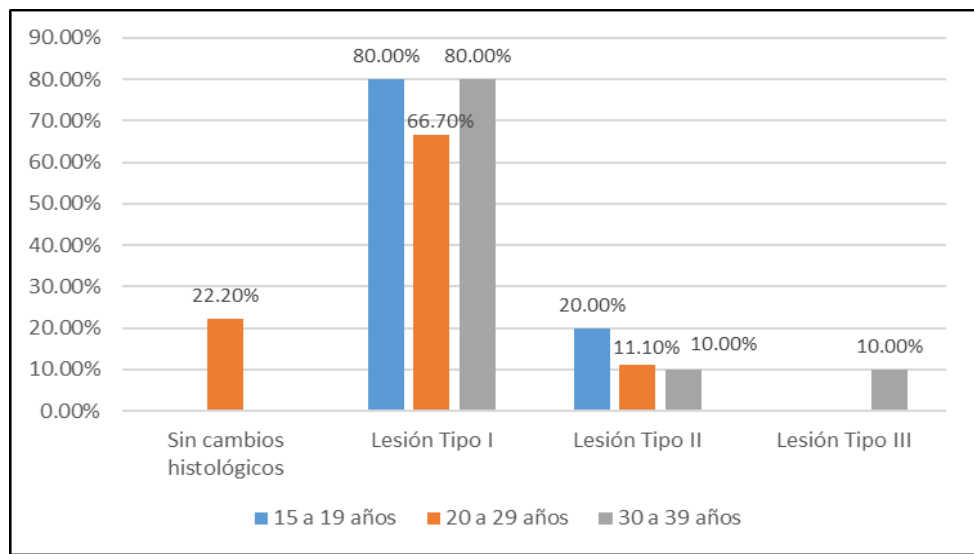

Figura 6. Frecuencia de lesión en arteria coronaria según edad. 
En la gráfica 6 observamos que en el grupo de 15 a 19 años ninguna de las muestras presentaba coronarias sanas, un $80 \%$ manifestaba ya lesiones tipo I y un $20 \%$ lesiones tipo II. En el grupo de 2029 años, el 66.7\% presentaba lesiones tipo I y el 11.1\% lesiones tipo II. Cabe destacar que, en este grupo etario, un $22.2 \%$ no presentaban cambios histológicos, que probablemente pueda deberse a una distribución azarosa de la muestra. En el grupo de 30 a 39 años todos presentaron algún tipo de lesión, donde la mayor proporción, un $80 \%$ de todo el grupo representativo, presentaba una lesión tipo I y un 10\% presentaba una lesión tipo II o lesiones tipo III.

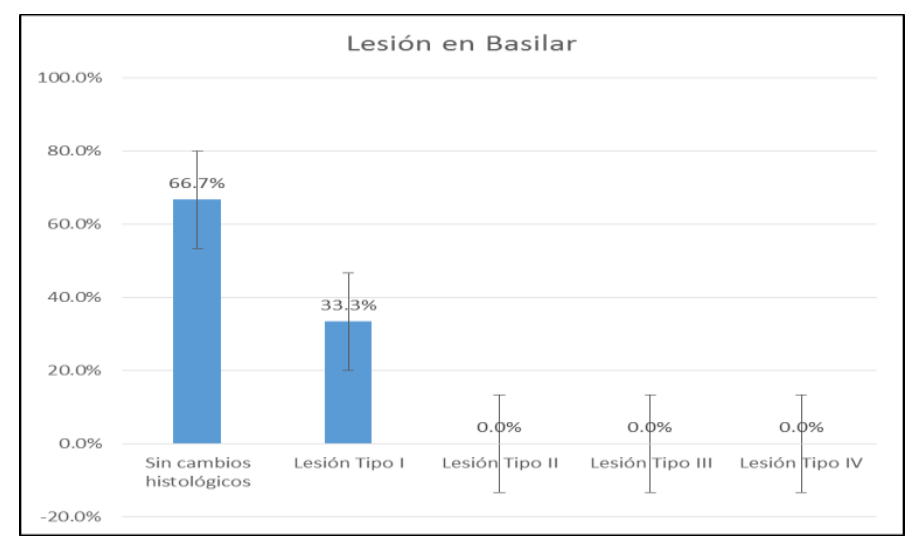

Figura 7. Frecuencia según tipo de lesión en arteria basilar

El $66.7 \%$ de las muestras no tuvieron cambios histológicos a nivel de arteria basilar. El 33.3\% tuvo lesiones tipo I. (Graf. 7).

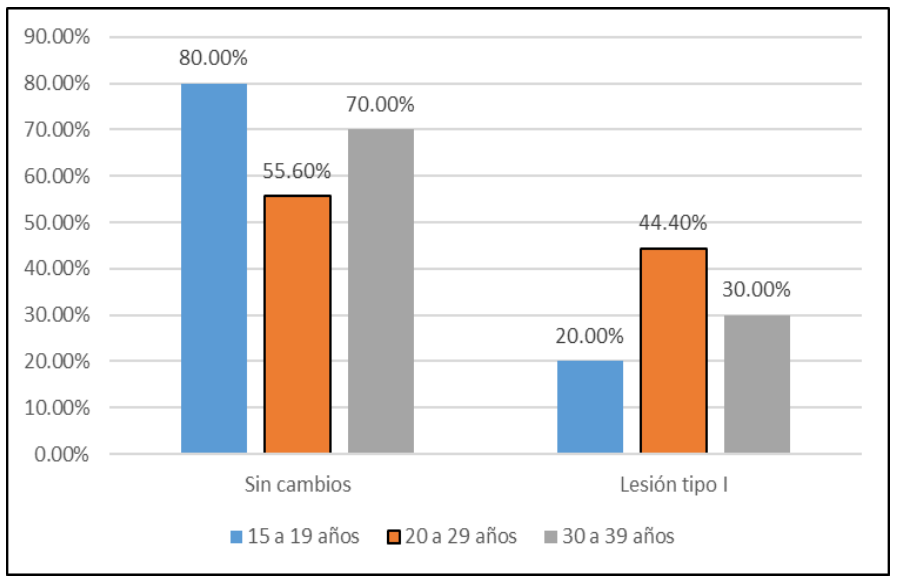

Figura 8. Frecuencia de lesión en arteria basilar según edad.

En los tres grupos de edad observados las lesiones llegaron a ser principalmente de tipo I, $20 \%$ en el grupo de 15 a 19 años, $44.4 \%$ en el grupo de 20 a 29 años y de $30 \%$ en el grupo de 30 a 39 años.

En general, las lesiones tipo I se observaron más en coronaria (72\%), las de tipo II coronaria y carótida (12\%), tipo III en aorta (8\%) y tipo IV en aorta (8\%). 


\section{DISCUSIÓN}

La arteriosclerosis es un término genérico que se refiere al engrosamiento y el endurecimiento de las arterias, independientemente de su tamaño. Su lesión básica es la placa de ateroma compuesta fundamentalmente de lípidos, tejido fibroso y células inflamatorias, y pasa por diferentes estadios (5) Es sistémica y tiene como blancos principales las arterias coronarias, carótidas (vertebrales y cerebrales) y las extremidades inferiores (iliacas y femorales) (6) Una de las etiologías en estudio es que existe un importante sustrato de enfermedad inflamatoria, con un cuerpo integrado de mediadores químicos y celulares, los cuales, como vía final, conducen al daño (apoptosis) de los macrófagos tras la imposibilidad por parte del sistema de reparar las lesiones(7). La Calcificación vascular es un sello del aterosclerosis. La localización, densidad y confluencia de la calcificación puede cambiar en porciones del conducto arterial. La microcalcificación en la cápsula fibrosa puede aumentar el daño del tejido (dependiendo de la proximidad de un locus microcalcificó a otro, y la orientación de la microcalcificación en referencia al flujo sanguíneo), resultando en inestabilidad de placa (8).

La presente investigación crea la necesidad de investigar el estado sanitario de las personas, especialmente jóvenes, de nuestro entorno regional, a través de la tipificación de daño de las arterias. Este marcador es un indicador indirecto de los estilos de vida de una población, así como el impacto real de las estrategias preventivas que pudiesen existir para mitigar el problema o controlarlo. Es absolutamente conocido que el estilo dietético de nuestra sociedad está basado básicamente en el consumo de grasas saturadas de origen animal, así como el consumo de elementos de fabricación industrial procesado que se añade a lo anteriormente referido. No existe un programa real preventivo de cultura sanitaria ni alimentaria. Agrava el problema el saber que no hay trabajos de investigación que midan el impacto de daño a través de resultados tangibles de cómo se encuentra el estado real de nuestra población asociado a costumbres de estilos de vida o estilos dietéticos. Realizamos una investigación, que, por los limitados recursos, nos permitían trabajar con muestras post mortem de personas y registradas en el sistema como residentes en la región. El estudio de las arterias basilar, carótida, coronaria y aorta, son indicadores trazadores de daño actual y predictores indirectos de estilos de vida alimentario y sanitario en general. El estado de salud del ser humano es "la salud en el que se encuentran sus arterias".

Finney AC et al, en el 2017, refieren que para limitar las enfermedades cardiovasculares y sus complicaciones es necesario que siempre el daño sea diagnosticado oportunamente y en edades tempranas.(9). Trabajamos con muestras procedentes de personas entre 15 a 35 años, con el objetivo de demostrar que el daño arterial en este medio empieza desde muy temprano. Utilizamos arterias centrales coincidente a la propuesta de Wei B, Qian et al en el 2016 quien en una revisión sistemática encontraron que la enfermedad de las arterias periféricas asociadas significativamente a aumentos en la mortalidad por causa cardiovascular, concluyeron que la enfermedad de las arterias se asocia con un peor pronóstico general en cualquier paciente y concluye que hay que prestar atención a la arteriosclerosis especialmente de aparición en gente a edades tempranas.(10). Incluso Inglis SC et al, en el 2013, refieren que la enfermedad arterial a menudo pasa desapercibida, y sin embargo, a pesar de la disponibilidad de herramientas de detección modernas, esto no es comúnmente considerado en la atención médica por una inadecuada planificación sanitaria (11). El uso de enfermedad arterial periférica como marcador de riesgo para diferentes enfermedades subyacentes puede mejorar la eficacia de los criterios de selección para la 
detección en edades tempranas.(2). Sampaio et al, verifica la incidencia de obstrucciones ateroscleróticas y lesiones ateroscleróticas microscópicas en arterias radiales diseccionadas de cadáveres de más de 34 años (3) y considera la herramienta de trabajo post mortem como un adecuado trazador. Futuras investigaciones en nuestra región deben considerar la probabilidad de hacer el estudio en arterias periféricas y comparar el daño con arterias centrales como las estudiadas. Estimar la posibilidad de encontrar una relación que estadísticamente pueda ser relacionada. Obtener una muestra periférica en el futuro podría ser más sencillo y así poder estimar el estado de arterias centrales. Esta estrategia especialmente es posible puesto que, en sociedades donde el recurso de diagnóstico por imágenes es limitado, se presenta como una herramienta accesible y más exacta.

Van Engelen at el, en el 2013 refiere que las secciones histológicas proporcionan información precisa sobre la composición de la placa aterosclerótica y se usan en diversas aplicaciones como los estudios de prevalencia poblacionales o regionales. Afirma se pueden obtener buenas segmentaciones histológicas mediante segmentación en muestras de fallecidos. Además, estos pueden ser utilizados para desarrollar métodos de segmentación y visualización que permitan mejorar diagnósticos.(12). Ponte-Negretti Cl y at el, en el 2017 expone un resumen ejecutivo elaborado por un grupo de expertos llamado Academia Latinoamericana para el Estudio de los Lípidos (ALALIP). Un análisis sistemático de las encuestas nacionales de salud y los estudios de cohortes regionales mostraron una prevalencia consistentemente alta de las anomalías lipídicas que definen la arteriosclerosis. Refiere que estas anormalidades podrían estar relacionadas con el alto consumo de alimentos con una alta densidad calórica, colesterol y grasas trans, un estilo de vida sedentario y quizás cambios epigenéticos. Esto necesariamente estará muy relacionado a problemas de ateromatosis en arterias desde temprana edad (4). Károlyi $\mathrm{M}$ y at el, en el 2013 buscó determinar si la clasificación de las placas ateroscleróticas coronarias humanas con T1, T2 y resonancia magnética cardíaca (CMR) se correlacionaría bien con la clasificación de la placa aterosclerótica por histología. Lograron determinar que el uso de resonancia es lo más cercano a la precisión del diagnóstico corroborado por histología cuando se trabaja en vivos(13). Esto dependerá de los recursos existentes en cada sociedad humana. Jiang Y at el, en el 2016, refiere que el desarrollo avanzado de técnicas de Resonancia Magnética de alta resolución ha permitido la obtención de imágenes de placa aterosclerótica intracraneal in vivo. Sin embargo, la identificación de la composición de la placa sigue siendo desafiante dado el pequeño tamaño y la falta de validación histológica.(14) Lastimosamente estos estudios no son de uso común en nuestro medio y región que precisa estudios primarios post mortem para determinar primariamente el daño, hasta encontrar mejores instrumentos.

Nakahara $T$ et al, revisaron literatura relevante mediante búsquedas de estudios publicados y afirman que las calcificaciones también pueden formar el capuchón de un fibroateroma de capa delgada, alterando las fuerzas de tracción sobre la tapa y haciendo que la lesión sea propensa a la ruptura. Refiere que muchos factores locales y sistémicos participan en este proceso, incluyendo hiperlipidemia, inflamación en curso, núcleos necróticos grandes y diabetes. La calcificación coronaria es un marcador de ateroma coronario, la calcificación densa (> $400 \mathrm{HU}$ ) suele asociarse con placas estables(8). Es necesario realizar posteriores investigaciones en la histopatología de las arterias propuestas en la siguiente investigación. 
La tipificación de calcificaciones o microcalcificaciones en arterias especialmente de sistema nerviosos central y cardiacas.

Con lo anteriormente referido nuestro deseo es sensibilizar para que se definan las estrategias de control sanitario y dietético, pero más aún proponer a la sociedad médica regional el estudio de las arterias como un marcador de estado sanitario poblacional.

Conflicto de interés: Los autores declaran la no existencia de conflictos de interés.

\section{Contribución de los autores:}

Dr. Victor Alfonso Arias Santana, Identificación del problema, concepción metodológica, análisis crítico y estadístico.

Mag. Pamela Cáceres Cáceres, clasificación de estructuras anatomopatológicas. Trabajo de campo, Mag. Sissy Soledad Mena Ordoñez. Análisis de datos, redacción y bibliografía.

Financiación: Universidad Privada de Tacna. Fondo Concursable. Artículo del informe final de la investigación entregado a la Universidad Privada de Tacna.

\section{REFERENCIAS BIBLIOGRÁFICAS}

1. OMS | Organización Mundial de la Salud [Internet]. WHO. [citado 30 de mayo de 2017]. Disponible en: http://www.who.int/es/

2. Anand RG, Ventura HO, Mehra MR. Is heart failure more prevalent in patients with peripheral arterial disease? A meta-analysis. Congest Heart Fail Greenwich Conn. diciembre de 2007; 13(6): 319-22.

3. Sampaio JAF de, Ferro MC, Alcoléa AP, Junior S, Da DC, Magna LA, et al. Incidência de aterosclerose em artérias radiais de cadáveres. Rev Bras Cir Cardiovasc [Internet]. 200606 [citado 30 de mayo de 2017]; 21(2): 165-72. Disponible en:

http://www.scielo.br/scielo.php?script=sci_arttext\&pid=S1678-97412006000200009

4. Ponte-Negretti $\mathrm{Cl}$. Atherogenic dyslipidemia in Latin America: Prevalence, causes and treatment: Expert's position paper made by the Latin American Academy for the study of Lipids (ALALIP) endorsed by the Inter-American Society of Cardiology (IASC), the South American Society of Cardiology (SSC), the Pan-American College of Endothelium (PACE), and the International Atherosclerosis Society (IAS). Int J Cardiol. 18 de mayo de 2017;

5. Stary HC, Chandler AB, Dinsmore RE, Fuster V, Glagov S, Insull W, et al. A definition of advanced types of atherosclerotic lesions and a histological classification of atherosclerosis. A report from the Committee on Vascular Lesions of the Council on Arteriosclerosis, American Heart Association. Circulation. 1 de septiembre de 1995; 92(5): 1355-74.

6. Viles-Gonzalez JF, Fuster V, Badimon JJ. Atherothrombosis: a widespread disease with unpredictable and life-threatening consequences. Eur Heart J. julio de 2004;25(14):1197-207.

7. Fuster $\mathrm{V}$, Badimon L, Badimon JJ, Chesebro JH. The pathogenesis of coronary artery disease and the acute coronary syndromes (2). N Engl J Med. 30 de enero de 1992;326(5):310-8.

8. Nakahara T, Dweck MR, Narula N, Pisapia D, Narula J, Strauss HW. Coronary Artery Calcification: From Mechanism to Molecular Imaging. JACC Cardiovasc Imaging. mayo de 2017;10(5):582-93.

9. Finney $A C$, Stokes $K Y$, Pattillo $C B$, Orr AW. Integrin signaling in atherosclerosis. Cell Mol Life Sci CMLS. junio de 2017; 74(12): 2263-82.

10. Wei B, Qian C, Fang Q, Wang Y. The Prognostic Value of Peripheral Artery Disease in Heart Failure: Insights from a Meta-analysis. Heart Lung Circ. diciembre de 2016;25(12):1195-202.

11. Inglis SC, Hermis A, Shehab S, Newton PJ, Lal S, Davidson PM. Peripheral arterial disease and chronic heart failure: a dangerous mix. Heart Fail Rev. julio de 2013; 18(4): 457-64. 
12. van Engelen A, Niessen WJ, Klein S, Groen HC, van Gaalen K, Verhagen HJ, et al. Automated segmentation of atherosclerotic histology based on pattern classification. J Pathol Inform. 2013; 4(Suppl): S3.

13. Károlyi $\mathrm{M}$, Seifarth $\mathrm{H}$, Liew $\mathrm{G}$, Schlett $\mathrm{CL}$, Maurovich-Horvat $\mathrm{P}$, Stolzmann $\mathrm{P}$, et al. Classification of coronary atherosclerotic plaques ex vivo with $\mathrm{T} 1, \mathrm{~T} 2$, and ultrashort echo time CMR. JACC Cardiovasc Imaging. abril de 2013; 6 (4): 466-74.

14. Jiang $Y$, Zhu C, Peng W, Degnan AJ, Chen L, Wang $X$, et al. Ex-vivo imaging and plaque type classification of intracranial atherosclerotic plaque using high resolution MRI. Atherosclerosis. junio de 2016; 249: 10-6.

15. Perez ED, Hecht GM, Harwicz PS, Zimmerman EH. Ateromatosis aortica: un indicador de riesgo cardiovascular. Rev Argent Cardiol. 2000; 68:7. 